\title{
Chern-Simons term in the 4-dimensional SU(2) Higgs Model
}

\author{
F. Karsch ${ }^{a}$ b , M. L. Laursen ${ }^{a}$, T. Neuhaus ${ }^{b}$ and B. Plache ${ }^{b}$ \\ ${ }^{a}$ HLRZ, c/o KFA Jülich, P.O. Box 1913, D-5170 Jülich, Germany \\ ${ }^{b}$ Fak. f. Physik, Univ. Bielefeld, D-4800 Bielefeld, Germany
}

Using Seibergs definition for the geometric charge in SU(2) lattice gauge theory, we have managed to apply it also to the Chern-Simons term. We checked the periodic structure and determined the Chern-Simons density on small lattices $L^{4}$ and $L^{3} \times 2,4$ with $L=4,6$, and 8 near the critical region in the SU(2) Higgs model. The data indicate that tunneling is increased at high temperature.

\section{Introduction}

Some years back 't Hooft found that the baryon number and the lepton number are not conserved in the electroweak theory [1]. While the $B-L$ symmetry remains unbroken due to the anomaly cancellation, $B+L$ is no longer conserved. This socalled baryon number violation is caused by the nontrivial topological winding of the $\mathrm{SU}(2)$ gauge fields. The anomaly of the fermionic current relates the winding of the gauge fields and changes the baryon number by an amount

$B\left(t_{2}\right)-B\left(t_{1}\right)=\frac{N_{f}}{16 \pi^{2}} \int_{t_{1}}^{t_{2}} \int d^{3} x \operatorname{tr}\left[F_{\mu \nu} \tilde{F}_{\mu \nu}\right]$

where $N_{f}$ is the number of families of quarks and leptons. In the axial gauge $A_{0}=0$ we can relate the change in the baryon number to the change in the Chern-Simons number

$B\left(t_{2}\right)-B\left(t_{1}\right)=N_{f}\left[N_{C S}\left(t_{2}\right)-N_{C S}\left(t_{1}\right)\right]$

where the Chern-Simons number $N_{C S}$ is

$N_{C S}=-\frac{1}{8 \pi^{2}} \int d^{3} x \epsilon_{i j k} \operatorname{tr}\left[A_{i}\left(\partial_{j} A_{k}+\frac{2}{3} A_{j} A_{k}\right)\right]$.

At zero temperature such processes are exponentially suppressed as $\exp \left(-2 \pi / \alpha_{W}\right), \alpha \approx 1 / 30$. This is because any gauge field configuration which changes the winding number has an action at least that of the barrier height $2 \pi / \alpha_{W}$.

At high temperatures which prevail in the early universe such an exponential suppression is absent, since the system can pass over the barrier

*Presented by M. L. Laursen classically. The only suppression factor is the Boltzmann factor $\exp (-\beta E)$ where $E$ is the barrier height, and this factor is close to one ref. [2]. Any baryon asymmetry generated at the GUT scale will get washed out as the universe approaches the electroweak phase transition from above. If one assumes that the transition is of first order, together with CP violating processes and thermal non-equilibrium (provided by the expansion of the universe) one can explain the baryon asymmetry in the universe.

There exists semiclassical solutions of the Yang-Mills Higgs fields which are believed to be important in this scenario. These solutions are known as sphalerons. They are static, have finite energy, but are unstable. The sphaleron sits so to speak on top of the barrier and it has a baryonic charge of $1 / 2$. While the instanton tunnels between two Chern-Simons vacua (in the axial gauge) one must imagine a different time dependent solution which interpolates between a Chern-Simons vacuum and the top of the barrier.

There are some lattice studies of baryon number violating processes in the $2 \mathrm{~d}$ - Abelian Higgs model [3], and in the 4d-SU(2) Higgs model [4]. The configurations are prepared at high temperature and the system is allowed to change via the classical Hamiltonian equation of motion. Since the axial gauge is used, the Gauss constraint must be implemented in addition. The Chern-Simons term $N_{C S}(t)$ is monitored during the time evolution as a function of the temperature. When the system passes through a sphaleron transition one finds $\Delta N_{C S}= \pm 1$. All these calculations are done in the real time formalism. 
We have initiated work on the $4 \mathrm{~d}-\mathrm{SU}(2)$ Higgs model in Euclidean time, trying to see how the temperature influences tunneling. To evaluate $N_{C S}$ we have used a geometric definition given by Seiberg see ref. [5]. It requires a two dimensional numerical integration, but we have an efficient vectorized code which is an improvement of the code used in ref. [6]. See also ref. [7] for alternative definitions of the Chern-Simons term.

\section{Topological charge and the Chern- Simons term in the continuum}

We will first define the topological charge in $\mathrm{SU}(2)$. The gauge field is $A_{\mu}$ and the gauge field tensor is $F_{\mu \nu}=\partial_{\mu} A_{\nu}-\partial_{\nu} A_{\mu}+\left[A_{\mu}, A_{\nu}\right]$. Under a a local gauge transformation $g$ the gauge field changes as: $\delta A_{\mu}=g^{-1}\left[A_{\mu}+\partial_{\mu}\right] g(x)$, while the gauge field tensor transforms gauge covariantly $F_{\mu \nu} \rightarrow g^{-1} F_{\mu \nu} g$. The topological charge $Q$ is gauge invariant and an integer,

$Q=-\frac{1}{32 \pi} \int_{M} d^{4} x \epsilon_{\mu \nu \rho \sigma} \operatorname{tr}\left[F_{\mu \nu} F_{\rho \sigma}\right] \in Z$.

The manifold is denoted $\mathrm{M}$ and we shall assume that its boundary $\partial M$ is a three sphere $S^{3}$. The topological charge density $q$ can be written as a perfect derivative

$q=-\frac{1}{32 \pi} \epsilon_{\mu \nu \rho \sigma} \operatorname{tr}\left[F_{\mu \nu} F_{\rho \sigma}\right]=\partial_{\mu} K_{\mu}$

where the Chern-Simons density $K_{\mu}$ is

$K_{\mu}=-\frac{1}{8 \pi^{2}} \epsilon_{\mu \nu \rho \sigma} \operatorname{tr}\left[A_{\nu}\left(\partial_{\rho} A_{\sigma}+\frac{2}{3} A_{\rho} A_{\sigma}\right)\right]$.

It is gauge variant and changes under the gauge transformation $g$ by an amount $\left(\mathcal{G}_{\nu}=\partial_{\nu} g g^{-1}\right)$

$$
\begin{aligned}
\delta K_{\mu}= & -\frac{1}{24 \pi^{2}} \epsilon_{\mu \nu \rho \sigma} \operatorname{tr}\left[\mathcal{G}_{\nu} \mathcal{G}_{\rho} \mathcal{G}_{\sigma}\right] \\
& -\frac{1}{8 \pi^{2}} \epsilon_{\mu \nu \rho \sigma} \partial_{\nu} \operatorname{tr}\left[\mathcal{G}_{\rho} A_{\sigma}\right]
\end{aligned}
$$

We define the (timelike) Chern-Simons number $N_{C S}$ as follows:

$N_{C S}=\int_{\partial M} d^{3} x K_{0} \notin Z$.

While $N_{C S}$ is only an integer for pure gauge configurations, the gauge variation is an integer (the boundary term vanishes) $\delta N_{C S}=-\frac{1}{24 \pi^{2}} \epsilon_{0 \nu \rho \sigma} \int_{\partial M} d^{3} x t r\left[\mathcal{G}_{\nu} \mathcal{G}_{\rho} \mathcal{G}_{\sigma}\right] \in Z$

This follows also from homotopy theory using the mapping $g: S^{3} \rightarrow S U(2)=S^{3}$. Such mappings are characterized with the homotopy class $\Pi_{3}\left(S^{3}\right) \in Z$.

\section{Topological charge and the Chern- Simons term on the lattice}

We will now consider the lattice version of the topological charge and the Chern-Simons number. We will use a geometric definition. Problems with dislocations will be ignored here. The manifold is a four torus $M=T^{4}$ and we will cover $M$ with cells (hypercubes) $c(n)$. Let the gauge potential $A_{\nu}^{n}$ be defined on $c(n)$ and likewise $A_{\nu}^{n-\hat{\mu}}$ be defined on $c(n-\hat{\mu})$. At the faces (cubes) $f(n, \mu)=c(n-\hat{\mu}) \cap c(n)$, we can relate the two potentials by a transition function $v_{n, \mu}$

$A_{\nu}^{n-\hat{\mu}}=v_{n, \mu}^{-1}\left[A_{\nu}^{n}+\partial_{\nu}\right] v_{n, \mu}$.

In Lüschers version one first fixes to a local complete axial gauge in each $c(n)$. This will define $v_{n, \mu}$ at the corners of the hypercube. It is then possible to extend it to the whole cube. The topological charge is $\left(\mathcal{S}_{\nu}=s^{-1} \partial_{\nu} s, \mathcal{P}_{\nu}=p^{-1} \partial_{\nu} p\right)$,

$$
\begin{aligned}
Q^{L}=\sum_{n} q^{L}(n) & =\sum_{n, \mu}(-1)^{\mu}\left(k_{n, \mu}-k_{n+\mu, \mu}\right) \\
(-1)^{\mu} k_{n, \mu}= & -\frac{1}{24 \pi^{2}} \epsilon_{\mu \nu \rho \sigma} \int_{f} d^{3} x \operatorname{tr}\left[\mathcal{S}_{\nu} \mathcal{S}_{\rho} \mathcal{S}_{\sigma}\right] \\
& +\frac{1}{8 \pi^{2}} \epsilon_{\mu \nu \rho \sigma} \int_{\partial f} d^{2} x \operatorname{tr}\left[\mathcal{P}_{\rho} \mathcal{S}_{o}\right](11)
\end{aligned}
$$

The function $s$ is defined on the cube, while $p$ is defined on the boundary of the cube. The actual expressions are given in ref. [5]. In Seibergs version no local gauge fixing is performed, but otherwise the same interpolation is performed. Replace $\left(s, p, k_{n, \mu}\right) \rightarrow\left(S, P, K_{n, \mu}\right)$. The difference is that that $S$ and $P$ only depend on the original gauge fields in the cube. Then $N_{C S}=\sum_{n}, K_{n_{\text {a }} \mu}$ is nothing but a Chern-Simons term (the summation is over the spatial lattice only). Like in the continuum it is only an integer for pure gauge 


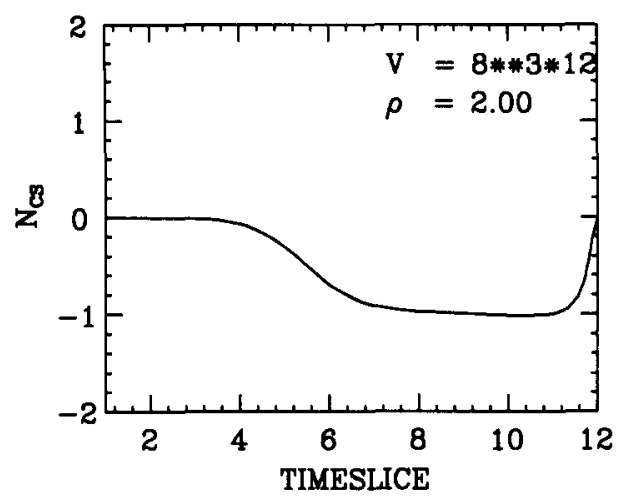

Figure 1. Profile of $N_{C S}$ through an instanton configuration.

field configurations, but under gauge transformations it changes by an integer. The corresponding topological charge is defined as $Q^{S}=\sum_{n} \tilde{q}^{S}(n)$, where $-1 / 2 \leq \tilde{q}^{S}(n)<1 / 2$. By restricting the charge to this interval we will have a gauge invariant charge definition. We find that both the topological charge and the Chern-Simons term have the correct naive continuum limit. As an interesting corollary we verify that the two charge definitions are related. If we introduce the section of the Lüscher bundle: $w(x), x \in \partial c(n)$, then $s(x)=w(0) S(x) w^{-1}(x)$ Inserting this in the above expressions yields $q^{S}(n)=q^{L}(n)-q^{w}(n)$ where $q^{w}(n)$ is the topological charge (integer) of the section. Therefore $\tilde{q}^{S}(n)=q^{L}(n)$ up to integers [8]. For smooth fields like instantons they always agree, while for realistic configurations this is true for almost every hypercube. It is interesting to look at the Chern-Simons number at each time slice of an instanton in the complete axial gauge. This is shown in Fig. 1. We start in the vacuum sector $N_{C S}=0$ and move towards the other vacuum sector $N_{C S}=1$. On the last time slice we must return to the first vacuum due to periodic boundary conditions.

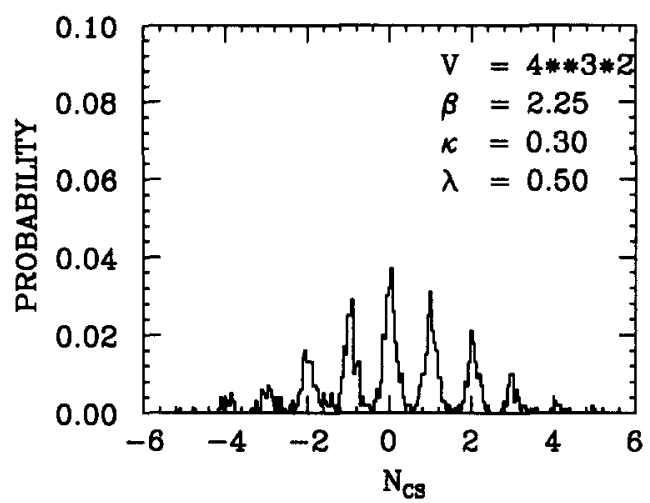

Figure 2. The periodic structure for $N_{C S}$.

\section{Tests and Monte-Carlo results for the Chern-Simons density}

We have used an SU(2) Higgs model with action:

$$
\begin{aligned}
& S=-\frac{\beta}{2} \sum_{n, \mu<\nu} \operatorname{tr}\left(U_{n, \mu \nu}\right)+\lambda \sum_{n}\left(\frac{1}{2} \operatorname{tr}\left(\Phi_{n}^{\dagger} \Phi_{n}\right)-1\right)^{2} \\
& -\kappa \sum_{n, \mu} \operatorname{tr}\left(\Phi_{n}^{\dagger} U_{n, \mu} \Phi_{n+\mu}\right)+\frac{1}{2} \sum_{n} \operatorname{tr}\left(\Phi_{n}^{\dagger} \Phi_{n}\right) .
\end{aligned}
$$

We always tried to work close to the Higgs phase transition. We looked at the periodic structure for a $4^{3} \times 2$ lattice at $(\beta, \kappa, \lambda)=(2.25,0.3,0.5)$. We did 1000 configurations and we measured $N_{C S}$ without any gauge fixing. In Fig. 2 we have plotted the Chern-Simons probability. The periodic structure is obvious. Notice that most of the configurations have $N_{C S}$ close to an integer, and therefore can be interpreted as being pure gauge. We checked that $N_{C S}$ indeed changed by an integer under an axial gauge transformation. Next we compared the probabilities for the two lattices $6^{4}$ and $6^{3} \times 2$. For these lattices we used 50 Landau gauge fixing sweeps, to make the integrals converge fast. In both cases we have 6000 Chern-Simons numbers. The results for the restricted and therefore gauge invariant $\tilde{N}_{C S}\left(-1 / 2 \leq \tilde{N}_{C S}<1 / 2\right)$ are shown in Fig. 3 and Fig. 4. There is a trend in the direc- 


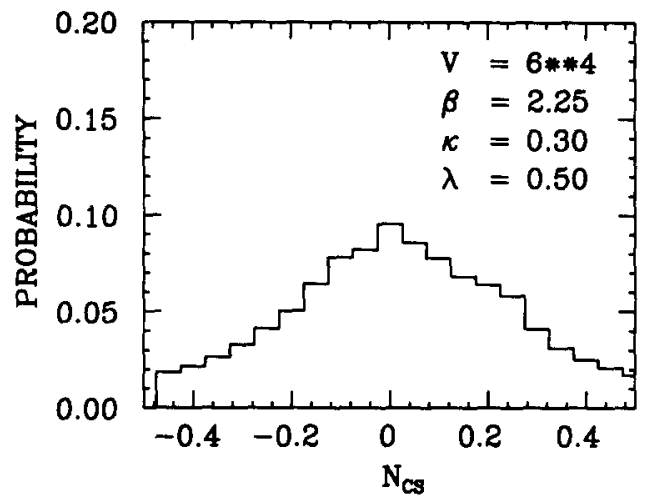

Figure 3. The Chern-Simons probability at zero temperature as a function of $\tilde{N}_{C S}$.

tion of a flatter distribution at finite temperature. We take this as evidence that the system tunnels more often. A few words about the integrations done. One of the integrations can be done analytically so we are left with a two dimensional integral. We have used the following strategy, which turned out to be quite efficient. Perform a Gaussian integration with $8 \times 8$ points and store the results for the eight $K_{n, \mu}$ 's in each hypercube. Redo the same thing with $16 \times 16$ points and compare the results for each $K_{n, \mu}$. If the relative difference is less than 0.0001 we accept the contribution. Otherwise we collect the $K_{n, \mu}$ 's which have not yet converged. These we redo with $32 \times 32$ points instead. Compare with the previous values and repeat the procedure with $64 \times 64$ points. Usually, at this point only a few $K_{n, \mu}$ 's have not converged, so for these we use a library integration routine with interval adaption. The typical time for one topological charge on a $6^{4}$ lattice is 100 seconds on the CRAY-YMP. The charges are integers up to errors of the order $10^{-4}$. The corresponding time for one Chern-Simons number in a timeslice is around 5 seconds.

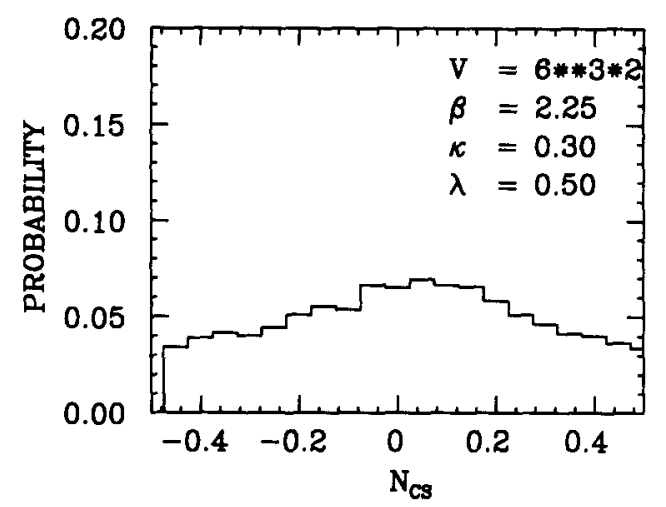

Figure 4. The Chern-Simons probability at finite temperature as a function of $\tilde{N}_{C S}$.

\section{REFERENCES}

1 G. 't Hooft, Phys. Rev. Lett. 37 (1976) 8; Phys. Rev. D14 (1976) 3432.

2 V. A. Kuzmin, V. A. Rubakov and M. E. Shaposhnikov, Phys. Lett. B155 (1985) 36.

3 D. Yu. Grigoriev, V. A. Rubakov and M. E. Shaposhnikov, Phys. Lett. B216 (1989) 172; Nucl. Phys. B326 (1989) 737;

A. I. Bochkarev and Ph. de Forcrand, Phys. Rev. D44 (1991) 519.

4 J. Ambjørn, M. L. Laursen and M. E. Shaposhnikov, Phys. Lett. B179 (1987) 757; Nucl. Phys. B353 (1989) 483; J. Ambjørn et al, Phys. Lett. B244 (1990) 479; J. Ambjørn, K. Farakos, NBI-HE-92-52 preprint.

5 M. Lüscher, Commun. Math. Phys.85 (1982) 39; N. Seiberg, Phys. Lett. B148 (1984) 456.

6 I. A. Fox, J. P. Gilchrist, M. L. Laursen and G. Schierholz, Phys. Rev. Lett.54 (1985) 749.

7 M. Göckeler, A. S. Kronfeld, G. Schierholz and U. -J. Wiese, HLRZ-92-34 preprint;

A. Phillips and D. Stone, Nucl. Phys, B (Proc. Suppl.) 20 (1991) 28.

8 M. L. Laursen, HLRZ-92-61 preprint. 